\title{
Peculiarities of the Clinical Picture and the Course of Acne and Rosacea at the Detection of Demodex Mites
}

\author{
Kubanov A, Gallyamova Y, Kravchenko A* \\ Russian Medical Academy of Continuous Professional Education of the Ministry of \\ Healthcare of the Russian Federation, Russia
}

*Corresponding author: Kravchenko Anzhela, Russian Medical Academy of Continuous Professional Education of the Ministry of Healthcare of the Russian Federation, 123995, 2/1 Barrikadnaya Street, Moscow, Russia, Tel: +44 7491814698; E-mail: angkravchenko@gmail.com
Received Date: August 08, 2017

Published Date: August 23, 2017

DOI: $10.23880 /$ cdoaj16000130

\section{Research Article \\ Volume 2 Issue 3}

\section{Abstract}

The article analyzes the clinical picture and course of acne and rosacea in patients with Demodex mites. The article presents the advantages of using the method of confocal laser scanning microscopy and the superiority of this method over the method of light microscopy of skin scrapings. It was found that Demodex mites complicate the course of acne and rosacea. Demodex folliculorum longus shows signs of parasitism, while Demodex folliculorum brevis is a saprophyte of facial skin. The severity of the disease does not depend on the quantitative load of the mites in the scraping. As an antiparasitic medication for the purpose of eliminating Demodex mites, it is recommended to use 7\% metronidazole in the form of a gel or cream-gel (depending on skin type) for 20 days externally.

Keywords: Dermatology life quality index; DLQI; Demodex mites; Demodex folliculorum longus; Demodex folliculorum brevis; Acne; Rosacea

\section{Introduction}

In connection with the growth of the social environment as a whole and the increase of individual exactingness to one's own appearance, both among men and women, dermatoses of the face remain one of the acute problems. Despite of a large number of scientific works devoted to the pathogenesis of acne and rosacea, the question concerning the role of Demodex mites in the development of the clinical picture of diseases remains open in modern literature.

According to some authors, Demodex mites are representatives of the opportunistic pathogenic microflora of the facial skin along with Propionbacterium acnes, Staphylococcus epidermidis and fungi of the genus Malassezia [1,2]. This opinion is supported by the fact that in $55-100 \%$ of cases, mites are detected, both in patients with facial dermatoses, and in individuals who do not have any clinical manifestations of dermatological diseases [3-5]. However, there are scientific works proving that Demodex mites are capable of pathogenic parasitizing and are the most frequently detected microbial agents in acne and rosacea [4]. At the same time, the absence of the diagnosis "Demodecosis" in the International Classification of Diseases X revision comes under notice, which points to the fact that Demodex mites act rather as an agent complicating the course of acne, rosacea and other acne-like dermatoses. Currently, two types of Demodex mites parasitize on human skin: Demodex folliculorum longus and Demodex folliculorum brevis [6]. In modern literature, there are no 


\section{Clinical Dermatology Open Access Journal}

substantiated scientific studies indicating the role of the species belonging of the causative agent in the formation of the clinical picture of acne and rosacea. Existing assumptions are not fully proven. The available data on the parasitizing of Demodex mites in patients with acne and rosacea are contradictory and, in many cases, mutually exclusive. Therefore, the detection of the peculiarities of the course of acne and rosacea in the presence of Demodex mites represents, on the one hand, great theoretical interest, on the other hand it has practical significance, determining new directions in the therapy of these diseases.

The available diagnostic methods for detecting Demodex mites do not meet the requirements of modern medicine; they do not guarantee the absolute reliability of the test results and are often traumatic. One of the modern diagnostic methods in dermatology is confocal laser scanning in vivo microscopy [7]. This is an innovative method, which advantages are noninvasiveness and high information content; however, to date this method has not been used to identify Demodex mites in the Russian Federation.

Thus, in order to improve the quality of diagnostics and therapy, it becomes necessary to conduct a scientific study with an analysis of the clinical picture, comparing the methods of diagnosis and treatment of patients with acne and rosacea associated with Demodex mites.

\section{The Study Goal}

To improve laboratory and instrumental diagnostics and therapy of patients with acne and rosacea associated with Demodex mites, taking into account clinical and diagnostic features.

\section{Objectives of the Study}

1. To study the influence of Demodex mites on the clinical picture and course of acne and rosacea.

2. To study the features of the clinical picture of acne and rosacea associated with Demodex mites, depending on the species belonging of Demodex mites.

3. Compare the effectiveness of laboratory and instrumental diagnostic methods for detecting Demodex mites.

4. To evaluate the effectiveness of external therapy aimed at eliminating Demodex mites in patients with acne and rosacea.

\section{Materials and Methods}

The work was carried out on the bases of the Department of Dermatovenereology and Cosmetology of the Federal State Budgetary Educational Institution of Further Professional Education "Russian Medical Academy of Continuing Professional Education" of the Ministry of Healthcare of the Russian Federation and the Federal State Budgetary Institution "State Scientific Center of Dermatovenereology and Cosmetology" of the Ministry of Healthcare of the Russian Federation from 2013 to 2016 years.

During the study, a total of 106 people (men and women) were examined. The study included healthy individuals, patients with diagnoses of acne and rosacea with the presence and absence of Demodex mites on the facial skin. During the study, three groups of patients and healthy persons over the age of 18 were formed. Group I patients with the diagnoses of rosacea and acne with the presence of Demodex mites. Group I included patients who have Demodex mites confirmed by two study methods (light microscopy of skin scrapings and confocal laser scanning in vivo microscopy in an amount of more than 5 individuals per $1 \mathrm{~cm}^{2}$ ). Group II is a comparison group made up of patients diagnosed with acne, rosacea without Demodex mites. Demodex mites were not found in patients from group II by two study methods. Group III is a comparison group, which included healthy individuals.

Study Methods: Collecting the anamnesis and questioning according to the questionnaire "Dermatology Life Quality Index," which included 10 points.

Clinical: Examination and establishment of a preliminary diagnosis. Diagnoses of "Acne" and "Rosacea" were established based on the clinical picture of the diseases. To assess the severity degree of acne, the classification of the European guidelines for the treatment of acne was used [8]. The severity degree of rosacea was assessed according to the classification of the National Rosacea Society [9]. All patients with the diagnoses of acne and rosacea were carried out the calculation of morthological elements on the whole surface of the facial skin.

\section{Laboratory:}

1. Definition of existence and species belonging of Demodex mites was performed by light microscopy of skin scrapes, contents of sebaceous glands, hair follicles of eyelashes and/ or eyebrows, calculation of the found 
individuals, larvae and eggs per unit area $\left(1 \mathrm{~cm}^{2}\right)$.

2. Determination of the $\mathrm{pH}$ level of the face skin.

\section{Instrumental:}

1. Photographing patients before and after treatment.

2. Examination of the facial skin with the help of confocal laser scanning in vivo microscopy to determine the presence of Demodex mites.

Statistical: Statistical data processing was carried out with the use of Microsoft Excel 2013 and SPSS 21 software. The relationship of categorical indicators was established with the use of Fisher's exact test. Fisher's exact test - is a test of statistical significance used in the analysis of categorical data when sample sizes are small. To assess the significance of differences in the size of the follicles was used one-way analysis of variance with paired comparisons. To assess the significance of differences in the absence of normal distribution MannWhitney criteria and Kruskal-Wallis test for multiple comparisons were used. Differences were considered significant at $p<0.05$. The design of the study is shown in Figure 1.

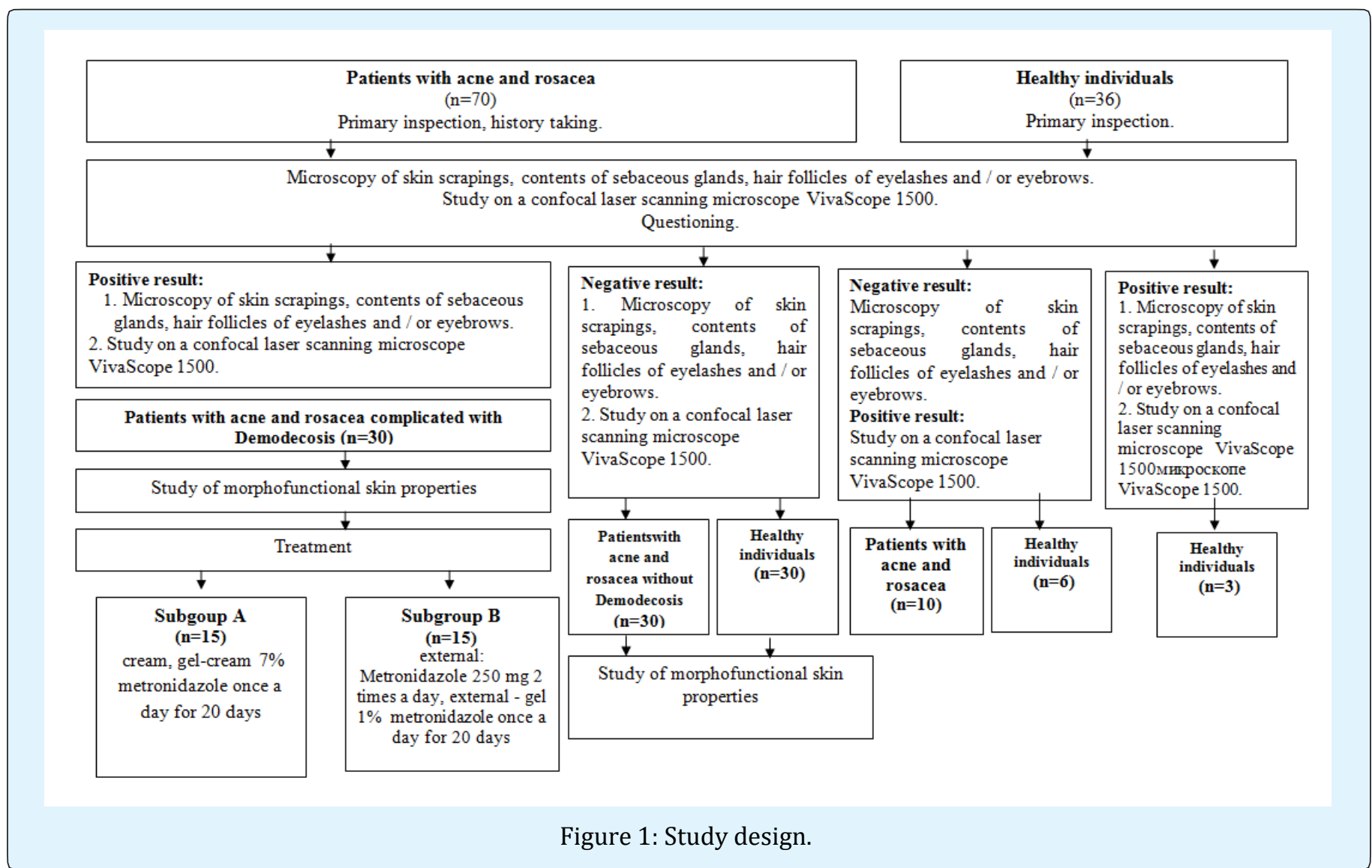

\section{Results}

\section{Clinical Characteristics of Patients Included in the Study}

During the study 106 respondents aged from 18 to 79 (39 men and 51 women) were under our supervision. The diagnosis of acne and rosacea has been exposed to 60 patients, among whom the diagnosis of acne had 40 persons (18 patients in group I and 22 in group II), the diagnosis of rosacea - $20 \quad(12$ and 8 patients, correspondingly) (Table 1).
According to the method of study, patients were divided into two groups. Group I included patients with the Demodex mites in number of more than 5 individuals on $1 \mathrm{~cm}^{2}$ which were detected by two study methods (light microscopy and confocal laser scanning in vivo microscopy) among them 12 patients with rosacea (40.0\%) and 18 patients with acne (60.0\%). Group II consisted of patients with a negative test for the presence of Demodex mites by two study methods (light microscopy and confocal laser scanning in vivo microscopy), among them - 22 patients with acne (73.3\%) and 8 patients with rosacea $(26.7 \%)$ (Table 1$)$. 


\begin{tabular}{|c|c|c|c|c|c|}
\hline \multicolumn{5}{|c|}{ I group (with the presence of Demodex mites) } & Total \\
\hline \multirow{2}{*}{ Sex } & \multirow{2}{*}{\multicolumn{2}{|c|}{$\begin{array}{c}\text { Men } \\
\mathrm{n}=12 ; 40.0 \%\end{array}$}} & \multirow{2}{*}{\multicolumn{2}{|c|}{$\begin{array}{c}\text { Women } \\
\mathrm{n}=18 ; 60.0 \%\end{array}$}} & \multirow{5}{*}{$30(100 \%)$} \\
\hline & & & & & \\
\hline \multirow{2}{*}{ Diagnose } & Acne & Rosacea & Acne & Rosacea & \\
\hline & $\mathrm{n}=8 ; 26.7 \%$ & $\mathrm{n}=4 ; 13.3 \%$ & $\mathrm{n}=10 ; 3.3 \%$ & $\mathrm{n}=8 ; 26.7 \%$ & \\
\hline Age & $23 \pm 5.5$ & $44 \pm 10.4$ & $29 \pm 6.4$ & $51 \pm 17.6$ & \\
\hline \multicolumn{5}{|c|}{ II group (with the absence of Demodex mites) } & \multirow{6}{*}{$30(100 \%)$} \\
\hline \multirow{2}{*}{ Sex } & \multirow{2}{*}{\multicolumn{2}{|c|}{$\frac{\text { Men }}{n=12 ; 40.0 \%}$}} & \multicolumn{2}{|c|}{ Women } & \\
\hline & & & \multicolumn{2}{|c|}{$\mathrm{n}=18 ; 60.0 \%$} & \\
\hline \multirow[b]{2}{*}{ Diagnose } & Acne & Rosacea & Acne & Rosacea & \\
\hline & $\mathrm{n}=9 ; 30.0 \%$ & $\mathrm{n}=3 ; 10.0 \%$ & $\mathrm{n}=13 ; 43.3 \%$ & $\mathrm{n}=5 ; 16.7 \%$ & \\
\hline Age & $24 \pm 2.2$ & $41 \pm 6.3$ & $33 \pm 2.9$ & $50 \pm 5.4$ & \\
\hline \multicolumn{5}{|c|}{ III group (healthy volunteers) } & \multirow{4}{*}{$30(100 \%)$} \\
\hline \multirow{2}{*}{ Sex } & \multicolumn{2}{|c|}{ Men } & \multicolumn{2}{|c|}{ Women } & \\
\hline & \multicolumn{2}{|c|}{$\mathrm{n}=15 ; 50 \%$} & \multicolumn{2}{|c|}{$\mathrm{n}=15 ; 50 \%$} & \\
\hline Age & \multicolumn{2}{|c|}{$26 \pm 1.4$} & \multicolumn{2}{|c|}{$31 \pm 3.1$} & \\
\hline
\end{tabular}

Table 1: Distribution of patients by sex, age, diagnosis and the presence of Demodex mites

Data analysis of the duration of the disease in groups was as follows (Table 2):

\begin{tabular}{|c|c|c|c|}
\hline Disease duration & I group & II group & $\begin{array}{c}\text { Total } \\
\text { (n; \%) }\end{array}$ \\
\hline (n; \%) $\mathbf{\%})$ & $3 ; 5$ & $12 ; 20 *$ & $15 ; 25$ \\
\hline 1 year 5 years & $5 ; 8.3$ & $12 ; 20 *$ & $17 ; 28.3$ \\
\hline More than 5 years & $22 ; 36.7^{*}$ & $6 ; 10$ & $28 ; 46.7$ \\
\hline Total & $30 ; 50$ & $30 ; 50$ & $60 ; 100$ \\
\hline
\end{tabular}

$*_{-} \mathrm{p}<0.01$

Table 2: Duration of acne and rosacea in patients of groups I and II.

According to the medical history, the majority of patients with acne and rosacea before the inclusion in the study received therapy for these diseases, being treated in dermatovenerologists, or by taking therapy alone (Table $3)$.

\begin{tabular}{|c|c|c|c|}
\hline & Primary treatment (n; \%) & Recue (n; \%) & Total (n; \%) \\
\hline I group & $8 ; 13.3$ & $22 ; 36.7^{*}$ & $30 ; 50$ \\
\hline II group & $14 ; 23.3$ & $16 ; 26.7$ & $30 ; 50$ \\
\hline Total & $22 ; 36.6$ & $38 ; 63.4$ & $60 ; 100$ \\
\hline
\end{tabular}

$*$ - $\mathrm{p}<0.01$

Table 3: Appealability of patients group I and II for dermatovenereological care regarding acne and rosacea.

Patients who turned repeatedly to the attendance were surveyed for the presence of acne and rosacea recurrence within a year and were divided into three groups: with one relapse per year, with relapses from one to three times per year and with relapses more than three times per year (Table 4). 


\begin{tabular}{|c|c|c|c|c|}
\hline & $\begin{array}{c}\text { 1 relapse } \\
\text { (n; \%) }\end{array}$ & $\begin{array}{c}\mathbf{1 - 3} \text { relapses } \\
\text { (n; \%) }\end{array}$ & $\begin{array}{c}\text { 3 and more relapses } \\
\text { (n; \%) }\end{array}$ & $\begin{array}{c}\text { Total } \\
\text { (n; \%) }\end{array}$ \\
\hline I group & $3 ; 7.9$ & $15 ; 39.5^{*}$ & $4 ; 10.5$ & $22 ; 57.9$ \\
\hline II group & $10 ; 26.3^{*}$ & $4 ; 10.5$ & $2 ; 5.3$ & $16 ; 42.1$ \\
\hline Total & $13 ; 34.2$ & $19 ; 50$ & $6 ; 15.8$ & $38 ; 100$ \\
\hline
\end{tabular}

$*-\mathrm{p}<0.05$

Table 4: The number of relapses of acne and rosacea at patients of groups I and II within a year.

\section{The Dermatology Life Quality Index Assessment} (DLQI)

When comparing average values of the dermatology life quality index statistically reliable difference was revealed. At patients of group I the average value of the index amounted to $12.5 \pm 4.5(\mathrm{~min}=5.0 ; \max =19.0)$. At patients of group II the average value of the dermatological index was equal to $12.5 \pm 4.5(\mathrm{~min}=5.0$; $\max =19.0)(\mathrm{p}<0.05)$. The average value of the dermatology life quality index with the presence of
Demodex folliculorum brevis is 10.5 . In the presence of Demodex folliculorum longus and combined cases of simultaneous detection of two species of mites - 15.5 and 13.5 respectively.

\section{Clinical Picture of Patients with Acne and Rosacea}

Distribution of patients according to the severity of acne and rosacea diseases is presented in Tables 5 and 6.

\begin{tabular}{|c|c|c|c|}
\hline $\begin{array}{c}\text { Disease severity } \\
\text { (degree) }\end{array}$ & $\begin{array}{c}\text { I group } \\
\text { (n; \%) }\end{array}$ & $\begin{array}{c}\text { II group } \\
\text { (n; \%) }\end{array}$ & $\begin{array}{c}\text { Total } \\
\text { (n; \%) }\end{array}$ \\
\hline I & $3 ; 7.5$ & $4 ; 10.0$ & $7 ; 17.5$ \\
\hline II & $2 ; 5.0$ & $12 ; 30^{*}$ & $14 ; 35.0$ \\
\hline III & $6 ; 15.0^{*}$ & $4 ; 10.0$ & $10 ; 25.0$ \\
\hline IV & $7 ; 17.5^{*}$ & $2 ; 5.0$ & $9 ; 22.5$ \\
\hline Total & $18 ; 45$ & $22 ; 55$ & $40 ; 100$ \\
\hline
\end{tabular}

$*-\mathrm{p}<0.01$

Table 5: Distribution of patients with acne of groups I and II according to the severity of disease.

\begin{tabular}{|c|c|c|c|}
\hline Disease form & $\begin{array}{c}\text { Group } \\
\text { (n; \%) }\end{array}$ & $\begin{array}{c}\text { II group } \\
\text { (n; \%) }\end{array}$ & $\begin{array}{c}\text { Total } \\
\text { (n; \%) }\end{array}$ \\
\hline Erythematous telangiectatic & $3 ; 15$ & $4 ; 20$ & $7 ; 35$ \\
\hline Papular & $2 ; 10$ & $3 ; 15$ & $5 ; 25$ \\
\hline Pustular & $2 ; 10^{*}$ & $1 ; 5$ & $3 ; 15$ \\
\hline Infiltrative-productive & $5 ; 25^{*}$ & 0 & $5 ; 25$ \\
\hline Total & $12 ; 60$ & $8 ; 40$ & $20 ; 100$ \\
\hline
\end{tabular}

$*-\mathrm{p}=0.05$

Table 6: Distribution of patients with rosacea of groups I and II according to the severity of disease

For the objectivity of the study patients with acne and rosacea from groups I and II were divided according to the severity of the clinical manifestations of the diseases (papules, pustules, open come dones, milium, telangiectasia, perifocal erythema, excoriation, pigmentation, greasy luster). Frequency of occurrence of various clinical manifestations of acne and rosacea is presented in Figure 2. 


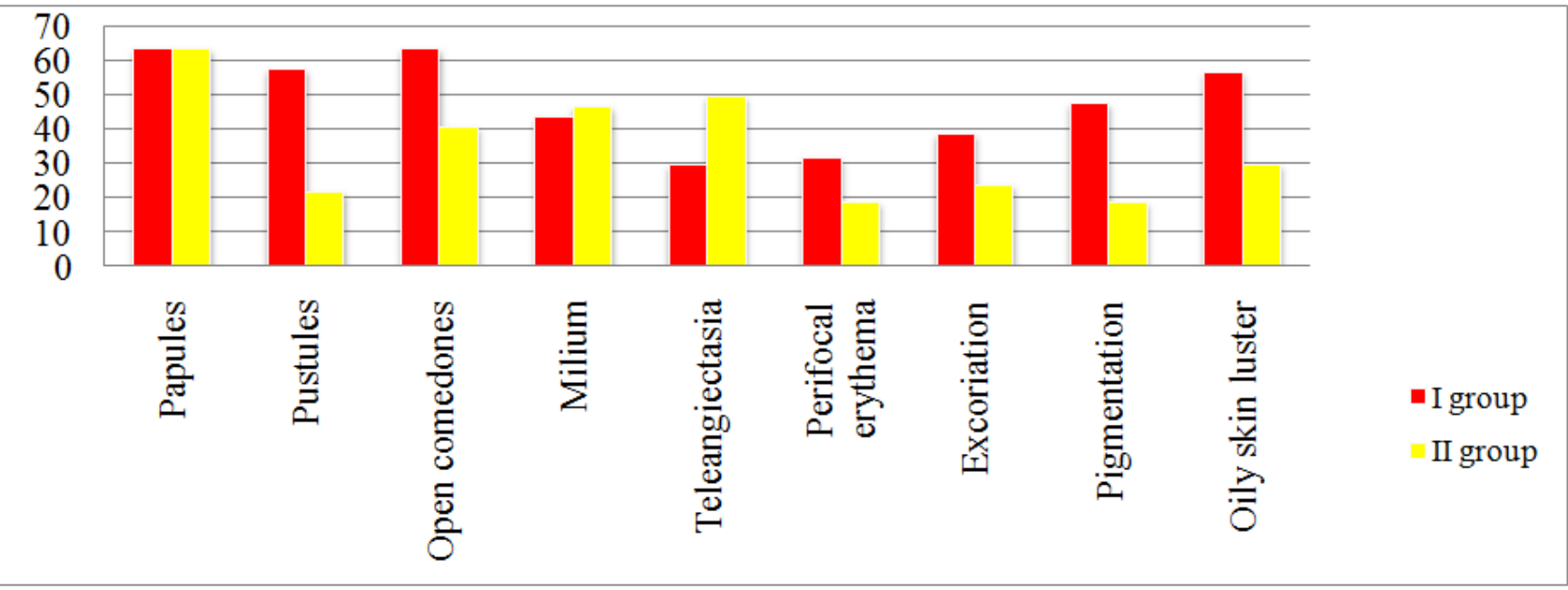

Figure 2: Frequency of occurrence of various clinical manifestations of acne and rosacea in patients from groups I and II.

\section{Determination of the Species Affiliation of Demodex Mites}

The largest number of cases of acne and rosacea at patients of group I was associated with parasitism of Demodex folliculorum longus $(\mathrm{n}=16 ; 53.3 \%)$, Demodex folliculorum brevis was detected in 9 patients (30\%), both species of mites were found in 5 patients (in $16.7 \%$ of cases).

At examining 36 healthy persons by light microscopy method, mites of the genus Demodex have been revealed in three cases $(n=3 ; 8.3 \%)$, in other 33 cases $(91.7 \%)$ we did not succeed to reveal mites. In determining the species belonging of Demodex mites in healthy individuals, the parasitism of Demodex folliculorum brevis was revealed in all three cases. In the structure of diagnoses of acne and rosacea Demodex folliculorum longus is significantly more prevalent $(\mathrm{n}=16 ; 53.3 \%)$. The quantity of cases of the detection of this type of mite prevails over the frequency of detection of Demodex folliculorum brevis and the combined parasitism by two species of mites.
The further study showed that Demodex folliculorum longus is accompanied by more severe forms of diseases (III-IV degree of acne, pustular and infiltrative-productive forms of rosacea) ( $\mathrm{n}=6$; and $33.3 \%, \mathrm{n}=5 ; 41.7 \%$, respectively). Combined cases of simultaneous detection of two species of mites on facial skin by light microscopy method (Demodex folliculorum longus and Demodex folliculorum brevis) also correlated with severe forms of acne and rosacea $(\mathrm{n}=3,16.7 \%$ and $\mathrm{n}=2 ; 16.7 \%$, respectively).

\section{Comparative Analysis of Diagnostic Methods for Detecting Demodex Mites}

To assess the accuracy of the confocal laser scanning in vivo microscopy method, the survey was conducted in all three groups of subjects (Figure 1). A comparative study was performed on the effectiveness of confocal laser scanning in vivo microscopy and scraping methods followed by microscopic examination. The obtained data are presented in table 7.

\begin{tabular}{|c|c|c|c|c|}
\hline Method & $\begin{array}{c}\text { Identification of Demodex mites }>5 \\
\left.\text { individuals per } 1 \mathrm{~cm}^{2}\right)\end{array}$ & $\begin{array}{c}\text { Patients with acne } \\
\text { and rosacea } \\
\text { diagnoses } \\
(\mathrm{n}=70 ; 66 \%)\end{array}$ & $\begin{array}{c}\text { Healthy } \\
\text { volunteers } \\
(\mathrm{n}=36 ; 34 \%)\end{array}$ & $\begin{array}{c}\text { Total }(\mathrm{n}=106 ; \\
100 \%)\end{array}$ \\
\hline $\begin{array}{c}\text { Scraping with subsequent } \\
\text { microscopy }\end{array}$ & + & $30 ; 28.3$ & $3 ; 2.8$ & $33 ; 31.1$ \\
\cline { 2 - 5 } & - & $40 ; 37.7$ & $33 ; 31.2$ & $73 ; 68.9$ \\
\hline
\end{tabular}




\begin{tabular}{|c|c|c|c|c|}
\hline Method & $\begin{array}{c}\text { Identification of Demodex mites }(>5 \\
\left.\text { individuals per } 1 \mathrm{~cm}^{2}\right)\end{array}$ & $\begin{array}{c}\text { Patients with acne } \\
\text { and rosacea } \\
\text { diagnoses } \\
(\mathrm{n}=70 ; 66 \%)\end{array}$ & $\begin{array}{c}\text { Healthy } \\
\text { volunteers } \\
(\mathrm{n}=36 ; 34 \%)\end{array}$ & $\begin{array}{c}\text { Total }(\mathrm{n}=106 ; \\
100 \%)\end{array}$ \\
\hline $\begin{array}{c}\text { Confocal laser scanning in } \\
\text { vivo microscopy }\end{array}$ & + & $40 ; 37.7$ & $6 ; 5.7$ & $46 ; 43.4$ \\
\cline { 2 - 5 } & - & $30 ; 28.3$ & $30 ; 28.3$ & $44 ; 56.6$ \\
\hline
\end{tabular}

Table 7: Comparative analysis of research methods for the presence of Demodex mites in diagnostically significant amounts.

30 patients with acne and rosacea (28.3\%) had a positive result for detecting Demodex mites by the light microscopy method of scrapings and 40 patients (37.7\%) by confocal laser scanning in vivo microscopy.

The method of confocal laser scanning in vivo microscopy also revealed Demodex mites in healthy individuals $(n=6,5.7 \%)$, and during the light microscopy of scrapings, Demodex mites in the number of 5 individuals per $1 \mathrm{~cm}^{2}$ were found only in three healthy persons $(n=3,2.8 \%)$. In the remaining 33 people $(31.2 \%)$, the data of microscopic diagnosis were negative.

\section{Quantification of Demodex Mites}

As a result of the study, we found that it is difficult to detect the mite by method of light microscopy of scrapings per $1 \mathrm{~cm}^{2}$ of skin (Table 8).

\begin{tabular}{|c|c|c|c|c|c|c|}
\hline $\begin{array}{l}\text { Patients with acne and } \\
\text { rosacea with the presence } \\
\text { of Demodex mites (n; \%) }\end{array}$ & \multicolumn{2}{|c|}{$\begin{array}{c}\text { Patients with acne and } \\
\text { rosacea without Demodex } \\
\text { mites } \\
(\mathrm{n} ; \%)\end{array}$} & \multicolumn{3}{|c|}{$\begin{array}{l}\text { Healthy volunteers } \\
\text { (n; \%) }\end{array}$} & \multirow[t]{2}{*}{ Total (n; \%) } \\
\hline $\begin{array}{l}\text { Presence of Demodex mites } \\
\qquad>5 / \mathrm{cm}^{2}\end{array}$ & $\begin{array}{c}\text { Presence of } \\
\text { Demodex mites } \\
<5 / \mathrm{CM}^{2}\end{array}$ & $\begin{array}{l}\text { Absence of } \\
\text { Demodex } \\
\text { mites }\end{array}$ & \begin{tabular}{|c|} 
Presence of \\
Demodex mites \\
$>5 / \mathrm{CM}^{2}$
\end{tabular} & \begin{tabular}{|c|} 
Presence of \\
Demodex mites \\
$<5 / \mathrm{CM}^{2}$
\end{tabular} & $\begin{array}{l}\text { Absence of } \\
\text { Demodex } \\
\text { mites }\end{array}$ & \\
\hline $30 ; 28.3$ & $11 ; 10.4$ & $29 ; 27.4$ & $1 ; 0.9$ & $2 ; 1.9$ & $33 ; 31.1$ & $106: 100$ \\
\hline $30 ; 28.3$ & \multicolumn{2}{|c|}{$40 ; 37.8$} & \multicolumn{3}{|c|}{$36 ; 33.9$} & \\
\hline
\end{tabular}

Table 8: Detection of Demodex mites by light microscopy of scrapings.

Demodex mites in a diagnostically significant amount (> 5 individuals per $1 \mathrm{~cm}^{2}$ ) were revealed in 30 patients with acne and rosacea (28.3\%), included in group I. In 40 patients with acne and rosacea (37.8\%), Demodex mites were detected in an amount of $<5$ individuals per $1 \mathrm{~cm}^{2}$ or were absent altogether with a developed clinical picture of the disease.

During the examination of healthy persons, 33 people (31.1\%) had a negative analysis for the presence of Demodex mites, while Demodex mites were detected in a diagnostic significant amount ( $>5$ individuals per $1 \mathrm{~cm}^{2}$ ) in one examined $(0.9 \%)$. In two persons, mites were found in an amount of $<5$ individuals per $1 \mathrm{~cm}^{2}(1.9 \%)$. Demodex mites in the number $<5$ individuals per $1 \mathrm{~cm}^{2}$ were detected much more often $(n=40,37.8 \%$, respectively).

When studing the same respondents using confocal laser scanning in vivo microscopy, the following data were obtained (Table 9)

\begin{tabular}{|c|c|c|c|c|}
\hline $\begin{array}{l}\text { Patients with acne and rosacea with the } \\
\text { presence of Demodex mites (n; \%) }\end{array}$ & $\begin{array}{l}\text { Patients with acne and rosacea } \\
\text { without Demodex mites (n; \%) }\end{array}$ & \multicolumn{2}{|c|}{ Healthy volunteers (n; \%) } & \multirow[b]{2}{*}{$\begin{array}{l}\text { Total } \\
(n ; \%)\end{array}$} \\
\hline Presence of Demodex mites $>5 / \mathrm{cm}^{2}$ & Absence of Demodex mites & $\begin{array}{c}\text { Presence of } \\
\text { Demodex mites } \\
>5 / \mathrm{cm}^{2}\end{array}$ & $\begin{array}{c}\text { Absence of } \\
\text { Demodex mites }\end{array}$ & \\
\hline $40 ; 37.8$ & $30 ; 28.3$ & $6 ; 5.7$ & $30 ; 28.3$ & \multirow{2}{*}{$106 ; 100$} \\
\hline $40 ; 37.8$ & $30 ; 28.3$ & \multicolumn{2}{|c|}{$36 ; 34$} & \\
\hline
\end{tabular}

Table 9: Detection of Demodex mites by confocal laser scanning in vivo microscopy. 
When analyzing the data in Table 9, attention is drawn to the fact that there were no $<5$ specimens of mites per 1 $\mathrm{cm}^{2}$ in any case.

By using confocal laser scanning in vivo microscopy, Demodex mites were found in patients with acne and rosacea $(\mathrm{n}=10,9.4 \%)$ and in healthy individuals in a larger number of cases $(n=6,5.7 \%)$.

Confocal laser scanning microscopy allows visualizing surface skin layers in vivo and getting extensional four dimensional images. The advantage of this method is high informational content, noninvasiveness and therefore, the absence of discomfort for patients. Demodex was defined as rounded or long cone-shaped formations in hair follicle orifices and sebaceous glands (Figure 3).

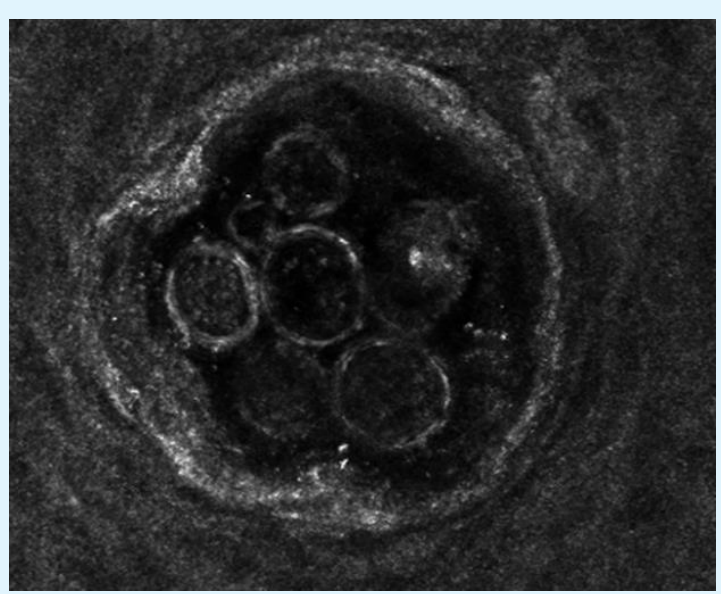

Figure 3: Images obtained using confocal laser scanning microscope VivaScope 1500®(Lucid Inc., Rochester, NY). Hair follicles and sebaceous glands with the presence of Demodex mites.

The use of confocal laser scanning in vivo microscope allowed determining the average size of Demodex mites. When determining the size of mites from 100 to $200 \mu \mathrm{m}$, it was believed that in this case Demodex folliculorum brevis was observed, while the average length of the mite was $125 \mu \mathrm{m}$; from 200 to $400 \mu \mathrm{m}$ - Demodex folliculorum longus with an average length of $293 \mu \mathrm{m}$. The average size of the Demodex mite width was $24 \mu \mathrm{m}$.

When measuring the size of the follicular entrance and estimating their number per unit area for which a randomly chosen site of $25 \mathrm{~mm} 2$ was taken, statistically significant differences between the three groups were revealed ( $p<0.01)$. It was established that the size of the follicular entrances and their number per unit area in all three groups differ significantly (Tables 10 \& 11).

\begin{tabular}{|c|c|c|}
\hline I group $(\boldsymbol{\mu m})$ & II group $(\boldsymbol{\mu m})$ & III group $(\boldsymbol{\mu m})$ \\
\hline $0.125 \pm 25$ & $0.89 \pm 32$ & $0.072 \pm 29$ \\
\hline
\end{tabular}

Table 10: The diameter of the follicle entrances and excretory ducts of the sebaceous glands in the study groups

\begin{tabular}{|c|c|c|}
\hline I group & II group & III group \\
\hline $324 \pm 11$ & $114 \pm 6$ & $28 \pm 7$ \\
\hline
\end{tabular}

Table 11: Number of follicles and excretory ducts of sebaceous glands in study groups per unit area $\left(25 \mathrm{~mm}^{2}\right)$

\section{Antiparasitic Therapy in Patients with Acne and Rosacea}

After obtaining a positive result of studies for the presence of Demodex mites, patients were randomized according to the treatment regimens into two equal subgroups (A and B, respectively) for 15 people each. Patients included in subgroup A received only external therapy with a medication containing $7 \%$ metronidazole in the form of a gel or cream gel once a day for 20 days. Patients included in subgroup $B$ received a medication containing $250 \mathrm{mg}$ of metronidazole systemically 2 times a day, externally $1 \%$ metronidazole in the form of a gel once a day for 20 days. A second visit of the patients took place after 20 days of continuous therapy. Subjectively, treatment regimens were tolerated well, no side effects were noted, non of the patients was excluded from the study.

When comparing the efficiency of the therapy, it was found that statistically significantly more Demodex mites were found after treatment with confocal laser scanning in vivo microscopy $(\mathrm{p} \leq 0.05)$ (Table 12).

\begin{tabular}{|l|c|c|c|c|}
\hline \multirow{2}{*}{} & \multicolumn{2}{|c|}{$\begin{array}{c}\text { Skin scraping, squeezing out the contents of the } \\
\text { sebaceous glands followed by microscopy }\end{array}$} & \multicolumn{2}{c|}{$\begin{array}{c}\text { Confocal laser scanning in vivo } \\
\text { microscopy }\end{array}$} \\
\cline { 2 - 5 } & Presence of Demodex mites & Absence of Demodex mites & $\begin{array}{c}\text { Presence of Demodex } \\
\text { mites Demodex }\end{array}$ & $\begin{array}{c}\text { Absence of } \\
\text { Demodex mites }\end{array}$ \\
\hline Subgroup A & $1 ; 3.3$ & $14 ; 46.7$ & $2 ; 6.7^{*}$ & $13 ; 43.3$ \\
\hline Subgroup B & $2 ; 6.7$ & $13 ; 43.3$ & $5 ; 16.7^{*}$ & $10 ; 33.3$ \\
\hline
\end{tabular}

* - $\mathrm{p} \leq 0.05$

Table 12: Comparative table of detection Demodex mites after the conducted treatment in subgroups A and B. 
For a comparative evaluation of the effectiveness of treatment methods in subgroups $A$ and $B$, in the next stage the clinical picture was compared in patients with acne and rosacea associated with Demodex mites before and after treatment. Patients complaints before and after the treatment are given in Table 13.

\begin{tabular}{|c|c|c|c|c|}
\hline \multirow{2}{*}{ Complains } & \multicolumn{2}{|c|}{ Subgroup A } & \multicolumn{2}{c|}{ Subgroup B } \\
\cline { 2 - 5 } & Before treatment & After treatment & Before treatment & After treatment \\
\hline Rash & $100 \%$ & $70 \%^{*}$ & $100 \%$ & $65 \%^{*}$ \\
\hline Pain & $68 \%$ & $34 \%^{*}$ & $75 \%$ & $30 \%^{*}$ \\
\hline Burning & $59 \%$ & $40 \%$ & $64 \%$ & $30 \%^{*}$ \\
\hline Redness & $69 \%$ & $40 \%^{*}$ & $70 \%$ & $40 \%^{*}$ \\
\hline Itching & $70 \%$ & $20 \%{ }^{*}$ & $54 \%$ & $10 \%^{*}$ \\
\hline Pigmentation & $42 \%$ & $25 \%$ & $36 \%$ & $17 \%$ \\
\hline Presence of crusts /excoriation & $45 \%$ & $30 \%$ & $39 \%$ & $20 \%$ \\
\hline Arterial spiders & $60 \%$ & $40 \%$ & $58 \%$ & $40 \%$ \\
\hline Oily skin luster & $70 \%$ & $40 \%{ }^{*}$ & $65 \%$ & $40 \%$ \\
\hline
\end{tabular}

$*-\mathrm{p} \leq 0.05$

Table 13: Comparison of subjective complaints of acne and rosacea patients associated with Demodex mites before and after treatment.

Analysis of the clinical picture of patients with acne after the therapy: (Figures $4 \& 5$ ). and rosacea associated with Demodex mites, before and

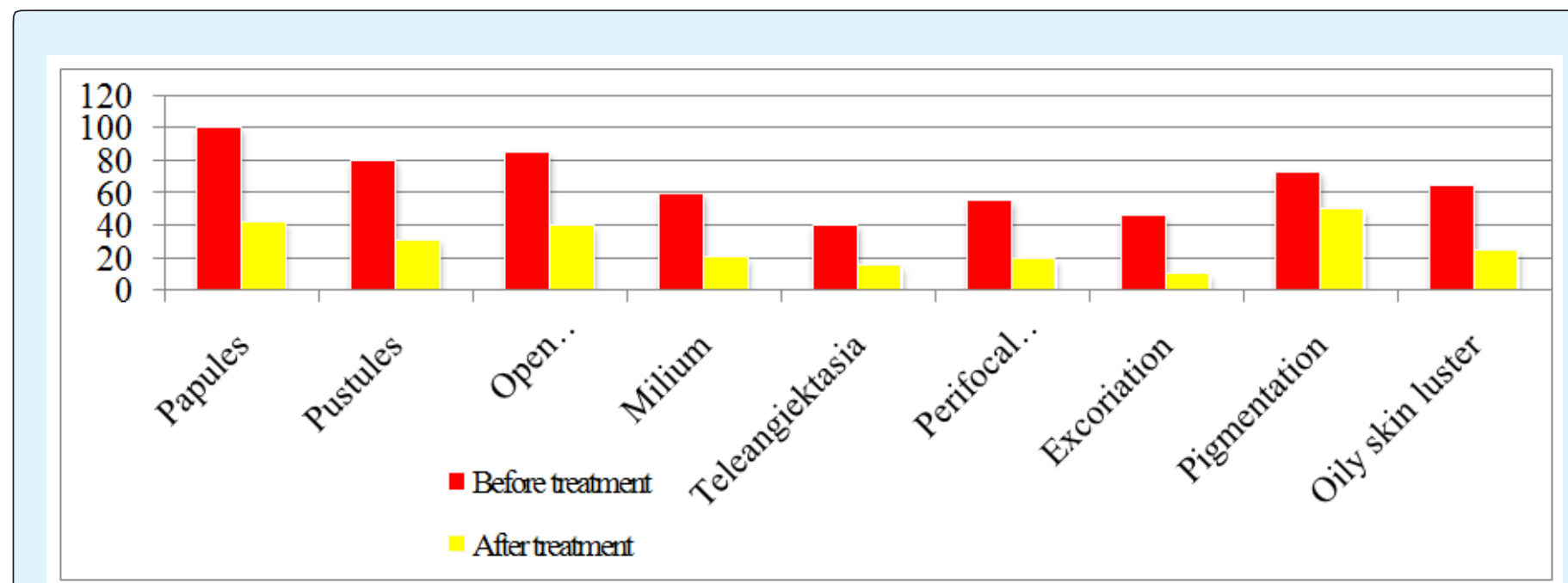

Figure 4: Frequency of occurrence of clinical manifestations of acne and rosacea before and after treatment in patients from subgroup A. 


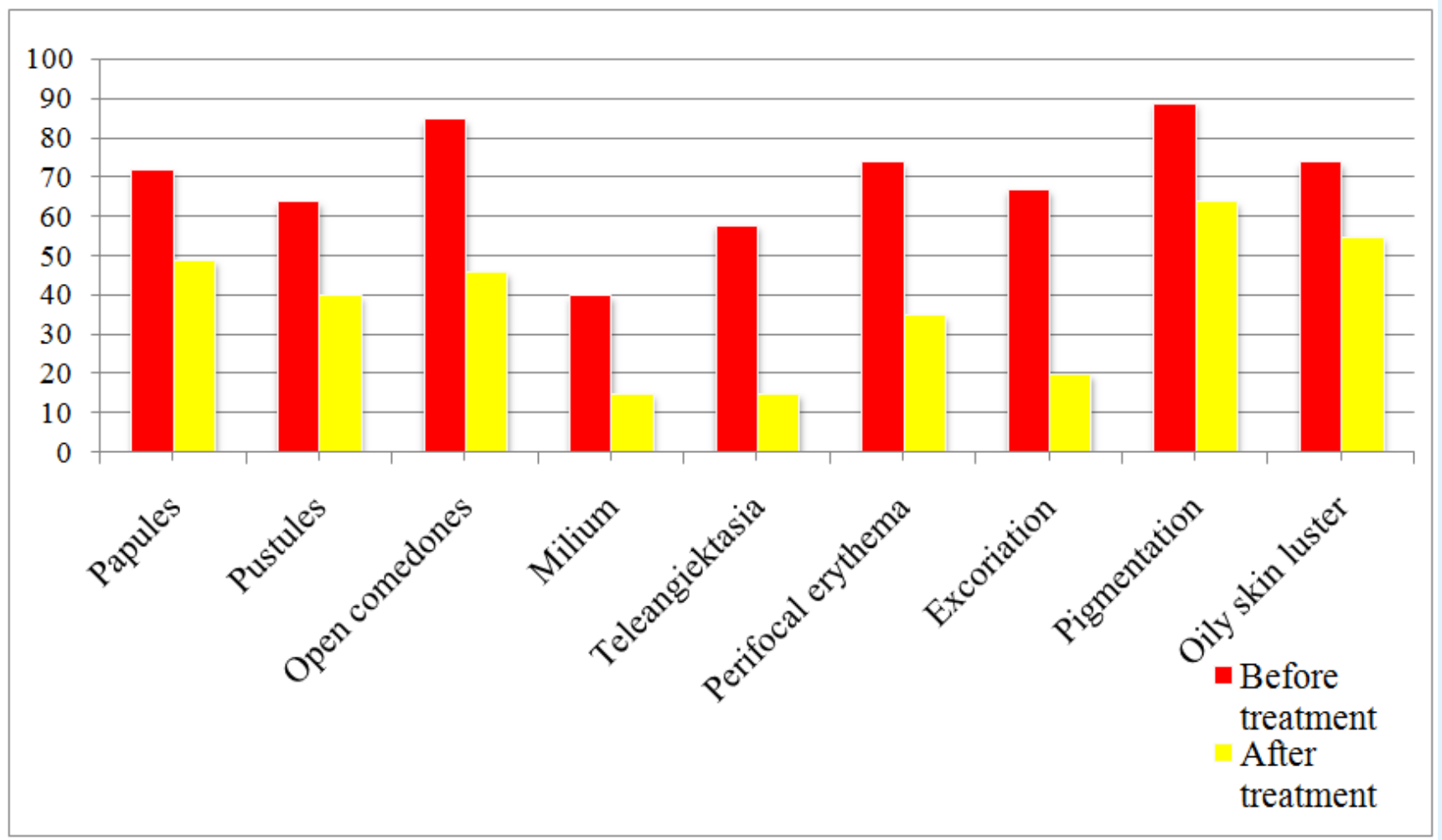

Figure 5: Frequency of occurrence of clinical manifestations of acne and rosacea before and after treatment in patients from subgroup B.

\section{Discussion}

When comparing the data of the anamnesis of groups I and II, we have established factors which are statistically significantly more frequent in patients with acne and rosacea associated with mites of the genus Demodex. Statistical processing of data revealed that the detection frequency of mites of the genus Demodex significantly differs depending on the factor provoking the development of the disease $(\mathrm{p}=0.001)$. All cases of mites detection were recorded with the following predisposing factors: emotional influences, including stress $(n=30$; $100 \%)$, inadequate nutrition $(\mathrm{n}=12 ; 40 \%)$, exacerbation of concomitant diseases $(n=1,3.3 \%)$. There were no statistical differences in the exacerbations of diseases, depending on the season and seasonality.

The data from table 2 demonstrate that among patients with acne and rosacea, which entered the group I and II, there are statistically significant differences $(\mathrm{p}<0.01)$, ie., the presence of mites of the genus Demodex in acne and rosacea contributes to a longer course of the diseases.
A statistically significant difference was found between the frequency of therapy in patients with acne and rosacea associated with mites of the genus Demodex (Table 3). Thus, we can come to a conlusion about the frequent recurrence of diseases in the presence of mites of the genus Demodex or the ineffectiveness of previous conducted therapy.

Comparing the frequency of recurrence of acne and rosacea between patients of groups I and II, there was revealed a statistically significant difference $(\mathrm{p}<0.05)$. In most cases, the disease recurred only once in patients without mites of the genus Demodex. Thus, mites of the genus Demodex complicating the clinical picture of the disease, promote a more frequent recurrence of the disease.

When comparing the average indicators of DLQI, it was revealed that in patients of group I the disease exerts a "very strong influence" on the life of the patient, in group II acne and rosacea had a "moderate effect" on the life of patients. 
Acne and rosacea complicated by the presence of Demodex folliculorum brevis have a "moderate impact" on the lives of patients. In the presence of Demodex folliculorum longus and combined cases of simultaneous detection of two species of mites, the disease "greatly affect" the lives of patients. Dermatological life quality index of patients with Demodex folliculorum is statistically significantly different from index of patients with Demodex folliculorum brevis ( $p<0.05$ ), which is logically natural, since we have found that Demodex folliculorum longus is detected in patients with more severe forms of the diseases.

As it is seen from the tables $5 \& 6$, mites of the genus Demodex were detected more frequently in patients with more severe clinical forms of acne (papulopustules, moderate nodular, severe nodular acne, acne conglobata) and rosacea (pustular and infiltrative-productive forms).

More severe clinical cases with the presence of deep papulopustules elements, nodules, perifocal erythema of the facial skin, oily luster prevailed in patients from group I ( $p<0.05)$ having Demodex mites. Patients from group II had papules, pustules, a small amount of telangiectasia and pigmentation.

When comparing clinical manifistations of the diseases the statistical difference between groups I and II was obtained. Such abnormal formations as deep papulopustules elements, nodules, facial skin erythema, oily luster are more pronounced in patients from group I, which once again confirms that the presence of Demodex mites contributes to the emergence of inflammatory elements and promotes the development of more severe clinical forms of diseases.

The data obtained in determining the species belonging of Demodex mites show that Demodex folliculorum longus is more reliably detected in the analyzes than Demodex folliculorum brevis, and Demodex folliculorum brevis is detected more often than the combined cases. Demodex folliculorum longus is statistically significantly more common in patients than simultaneous parasitizing of two species of mites ( $p<0.01)$. This suggests that, in the absence of clinical picture of acne and rosacea, it is significantly more important that Demodex mites will not be found in scrapings $(\mathrm{p}<0.01)$.

At analyzing the duration of the diseases, it was found that mites of Demodex folliculorum longus are statistically more frequently complicate acne and rosacea in patients of group I, resulting in disease course duration of about 5 years in comparison with Demodex folliculorum brevis and combined parasitizing of two species of mites ( $\mathrm{p} \leq$ 0.05). Diseases of acne and rosacea, accompanied by parasitism of Demodex folliculorum brevis were less lengthy and ranged from several months to one year $(\mathrm{p} \leq$ $0.05)$, which was statistically significantly lower than in the cases of association with Demodex folliculorum longus.

Comparing the results obtained by light microscopy and confocal laser scanning in vivo microscopy in patients with acne and rosacea and healthy individuals, in most cases Demodex mites are detected by confocal laser scanning in vivo microscopy, whereas scrapings in these patients were negative. The obtained data demonstrate not only high information content of the confocal laser scanning in vivo microscopy method, but also its superiority over microscopic diagnostics.

During the examination of healthy persons, 33 people (31.1\%) had a negative analysis for the presence of mites of the genus Demodex, while Demodex mites were detected in a diagnostic significant amount $>5$ individuals per $1 \mathrm{~cm}^{2}$ ) in one examined (0.9\%), two mites were found in an amount of $<5$ individuals per $1 \mathrm{~cm}^{2}$ (1.9\%). Considering the ability of mites to move along the surface of the skin at a speed of $8-16 \mathrm{~mm} / \mathrm{h}$, as well as random selection of the study site, this fact does not prove the absence mites $[10,11]$. Mites of the genus Demodex in the number $<5$ individuals per $1 \mathrm{~cm}^{2}$ were detected much more often $(n=40,37.8 \%$, respectively). Taking into account the species belonging of Demodex mites in healthy individuals (all had a Demodex folliculorum brevis), the absence of a clinical picture of the disease confirms that this species belongs to the saprophytes of facial skin.

By using confocal laser scanning in vivo microscopy, the mites of the genus Demodex were defined as rounded or long cone-shaped formations in the hair follicle orifices and sebaceous glands with peripheral contouring in the number from one to 25 individuals (an average of 3.37).

Obtaining alternately photos of all layers of the epidermis in steps of $3 \mu \mathrm{m}$, a confocal laser scanning in vivo microscopy allows to detected the mite at a depth of occurrence not accessible to scarification, such as for example in sebaceous gland ducts. The mite was recorded all over the epidermis. The average depth of the mites corresponds to the spinous layer of epidermis. Penetration of the parasite into the upper layers of the dermis was not revealed. 
When measuring the size of the follicular orificies and estimating their number per unit area, for which a randomly chosen site of $25 \mathrm{~mm}^{2}$ was taken, it was found that the largest size of the hair follicle orificies and the excretory ducts of the sebaceous glands had patients from group I with the presence of mites of the genus Demodex.

The comparison of the effectiveness of the conducted therapy once more demonstrates the superiority of the confocal laser scanning in vivo microscopy over the method of light microscopy of scrapings with further light microscopy (Table 12). At analizing the obtained data, given in table 12, we can conclude that both methods had high antiparasitic effectiveness. Considering the fact that there was no statistically significant difference in negative analyzes for the presence of mites of the genus Demodex in both laboratory and instrumental diagnostics, we can speak of a high antiparasitic efficiency of a topical medication containing 7\% metronidazole in comparison with combined treatment with the systemic drug metronidazole and a topical medication containing $1 \%$ metronidazole.

As can be seen from table 13, complaints of patients on rashes, burning, pain, itching and redness statistically reliably decreased after treatment. Moreover, in patients from subgroup A, complaints of oily skin gloss decreased, which is an additional advantage of topical therapy.

An objective analysis of the clinical picture of patients with acne and rosacea associated with Demodex mites after the conducted therapy showed that the following morphological elements in the subgroups $\mathrm{A}$ and $\mathrm{B}$ significantly regressed: papules, pustules, excoriations ( $p$ $=0.005$ ) in comparison with the initial data (Figures 4, 5).

Analysis of the clinical picture showed a positive dynamics of therapy, which manifested itself in a significant decrease in the number of morphological elements characterizing the severity of inflammation ( $p$ $<0.05$ ). The effectiveness of the therapy was confirmed by the reduction of subjective complaints of patients after the treatment, and patients who received only external therapy had no complaints of a feeling of skin oiliness and oily skin gloss, which is an additional advantage. After the antiparasitic therapy, the morphofunctional parameters of the skin were statistically improved $(p<0.05)$. Thus, clinical observations demonstrated a lack of superiority in combined antiparasitic therapy using a systemic drug, compared to external therapy using a medication containing $7 \%$ metronidazole in the form of a cream or cream-gel, as confirmed by statistical analysis.

\section{Conclusion}

1. Mites of the genus Demodex complicate the clinical picture and the course of acne and rosacea. When analyzing the clinical picture and the course of diseases in patients with acne and rosacea associated with mites of the genus Demodex, it is established that Demodex mites promote the development of acute-inflammatory morphological elements (deep papular and pustular elements, nodes, perifocal erythema), increase the duration of diseases (more than 5 years, $\mathrm{p}<0.01$ ) and the possibility of recurrence (from 1 to 3 relapses in $39.5 \%$ of patients, $\mathrm{p}<0.05$ ), resulting in a decrease in the quality of life of patients (DLQI $12.5 \pm 4.5, \mathrm{p}<0.05$ ). The severity of clinical manifestations of acne and rosacea does not depend on the number of detected individuals by light microscopy method of facial skin scrapings. The most significant factors predisposing to the development of complications are morphofunctional characteristics of the skin (high oiliness, reduced moisture, alkaline $\mathrm{pH}$ shift, larger pore size), emotional influence, including stress, ineffective nutrition and exacerbation of concomitant diseases.

2. In patients with severe manifestations of diseases (III, IV degree of acne, pustular and infiltrative-productive forms of rosacea), the species of Demodex folliculorum longus ( $p<0.01$ ) is more often found. Demodex folliculorum brevis is found in mild forms of diseases and in healthy individuals, without showing signs of parasitism $(\mathrm{p}<0.01)$.

3. Confocal laser scanning in vivo microscopy is an effective method for diagnosing mites of the genus Demodex that does not require preliminary preparation for analysis. This method allows to detect Demodex mites at the level of the spineous epidermis layer, which is not accessible for scarification, to identify the species belonging according to the size of Demodex mites (from 100 to $200 \mu \mathrm{m}$ - Demodex folliculorum brevis, 200 to $400 \mu \mathrm{m}$ - Demodex folliculorum longus).

4. The antiparasitic medication metronidazole, in the external form (gel, cream-gel), at a concentration of $7 \%$ ( 1 time per day, the general course of 20 days) has a high therapeutic efficiency in patients with acne and rosacea associated with mites of the genus Demodex (in $93.3 \%$ of cases). The effectiveness of the external therapy with a medication containing $7 \%$ metronidazole (20 days course) is comparable to the combined treatment with the systemic medication metronidazole $250 \mathrm{mg}$ per os twice a day and the 
external application of $1 \%$ metronidazole (gel) once a day for 20 days.

5. All patients with the diagnoses of "Acne (severe papulopustular and nodular acne of moderate and severe III, IV severity degrees)" and "postular and infiltrative-productive forms of rosacea" are prescribed a survey for the presence of mites of the genus Demodex with the definition of their species.

6. When Demodex folliculorum longus is detected, regardless of its quantitative load, treatment with antiparasitic medications is prescribed.

7. When Demodex folliculorum brevis is detected, taking into account its weak possibility of parasitism, treatment with antiparasitic medications is not prescribed.

8. As an antiparasitic medication for the purpose of eliminating mites of the genus Demodex, it is recommended to use 7\% metronidazole in the form of gel or cream-gel (depending on skin type) for 20 days externally. The drug should be applied a thin layer on the previously cleaned facial skin at night. The subsequent treatment should be carried out in accordance with the main diagnosis.

\section{References}

1. Lazaridou E, Giannopoulou C, Fotiadou C, Vakirlis E, Trigoni A, et al. (2011) The potential role of microorganisms in the development of rosacea. J Dtsch Dermatol Ges 9(1): 21-25.

2. Schommer NN,Gallo RL (2013) Structure and function of the human skin microbiome. Trends Microbiol 21(12): $660-668$.

3. Norn MS (1971) Demodex folliculorum. Incidence, regional distribution, pathogenicity. Dan Med Bull 18(1): 14-17.
4. Rufli T, Mumcuoglu Y (1981) The hair follicle mites Demodex folliculorum and Demodex brevis: Biology and medical importance. Dermatologica 162(1): 1-11.

5. Fujiwara S, Okubo Y,Irisawa R, Tsuboi R (2010) Rosaceiform dermatitis associated with topical tacrolimus treatment. J Am Acad Dermatol 62(6): 1050-1052.

6. Akbulatova LK (1966) The pathogenic role of Demodex mite and the clinical form of demodicosis in man. Vestn Dermatol Venerol; 40(12): 57-61.

7. Sattler EC, Maier T, Hoffmann VS, Hegyi J, Ruzicka T, et al. (2012) Noninvasive in vivo detection and quantification of Demodex mites by confocal laser scanning microscopy. Br J Dermatol 167(5): 10421047.

8. Nast A, Dreno B, Bettoli V, Degitz K, Erdmann R, et al. (2012) European Evidence-based (S3) Guidelines for the Treatment of Acne. J Eur Acad Dermatol Venereol 26(1): 1-29.

9. Wilkin J, Dahl M, Detmar M, Drake L, Feinstein A, et al. (2002) Standard classification of rosacea: Report of the National Rosacea Society Expert Committee on the Classification and Staging of Rosacea J Am Acad Dermatol 46(4): 584-587.

10. Hoekzema R, Hulsebosch HJ, Bos JD (1995) Demodecosis or rosacea: what did we treat? $\mathrm{Br} \mathrm{J}$ Dermatol 133(2): 294-299.

11. Demler M, de Kaspar HM (1997) J Ophthalmol 94(3): 191-193. 